\title{
Does the proportion of the brainstem change in the Parkinsonism?
}

\author{
Ilkay Camlidag ${ }^{\mathrm{a}}$, Mert Nahirb占, Fikri Ozdemir ${ }^{\mathrm{b}}$, Amani Elfaki ${ }^{\mathrm{b}}$, Murat Golpinar ${ }^{\mathrm{b}}$, Bunyamin Sahin $^{\mathrm{b}}$ \\ ${ }^{a}$ Deparment of Radiology, Faculty of Medicine, Ondokuz Mayis University, Samsun, Turkey \\ ${ }^{b}$ Deparment of Anatomy, Faculty of Medicine, Ondokuz Mayis University, Samsun, Turkey
}

\section{ARTICLE INFO}

\section{* Correspondence to:}

Mert Nahir

Deparment of Anatomy, Faculty of Medicine,

Ondokuz Mayis University,

Samsun, Turkey

e-mail: mert.nahir@gmail.com

\section{Keywords:}

Brainstem

Magnetic resonance imaging

Parkinson's disease

Planimetry

Volume fraction

\section{ABSTRACT}

Parkinson's disease (PD) is a slowly progressive neurological disorder that affects movement, muscle control, and balance. It develops as cells are destroyed in certain parts of the brain stem. The aim of this study is to measure the volume and volume fraction of the brainstem parts to examine the differences between affected regions in the PD. For the evaluation of brainstem volume, the sample comprised 18 Parkinson's patients ( 8 females and 10 males) in the $2^{\text {nd }}$ and $3^{\text {rd }}$ stages the disease, and 20 controls ( 9 females, 11 males). Magnetic resonance images in DICOM format were analyzed using the Image software. A protocol was developed to segment the parts of the brainstem and their volumes and volume fractions were estimated using the planimetry technique. The mean volume and volume fraction of the midbrain, pons, medulla oblongata and the total brainstem in control were $\left(8.2 \mathrm{~cm}^{3} \pm 1.14,27.61 \%\right),\left(16.25 \mathrm{~cm}^{3} \pm 2.75,54.37 \%\right),\left(5.32 \mathrm{~cm}^{3} \pm 0.73,18.02 \%\right)$, and $29.78 \mathrm{~cm}^{3} \pm 4.11$, respectively. And for patient groups were $\left(7.91 \mathrm{~cm}^{3} \pm 1.5,26.64 \%\right)$, $\left(16.25 \mathrm{~cm}^{3} \pm 2.49,55.38 \%\right),\left(5.32 \mathrm{~cm}^{3} \pm 0.89,17.98 \%\right)$, and $29.78 \mathrm{~cm}^{3} \pm 4.0$, respectively. There were no statistically significant differences between patients and controls for the volume and volume fraction of the brainstem parts $(\mathrm{p} \geq 0.05)$. Our data indicates that patients with PD do not show brainstem volume reduction in comparison with controls. Volumetric brain imaging studies in PD are conflicting with regards to brainstem atrophy. A number of factors may contribute to this variability, including differences in analysis methods, variability in the disorder itself and also due to variations in sampling selection. Our findings are still preliminary; we are trying to find changes associated with substantia nigra and red nucleus to evaluate the relation between the clinical scores of PD patients and imaging results.

J. Exp.Clin.Med., 2014; 31:145 